\title{
Critical and Topological Properties of Cluster Boundaries in the $3 d$ Ising Model
}

\author{
Vladimir S. Dotsenko[*] and Paul Windey \\ LPTHE[†] \\ Université Pierre et Marie Curie \\ Bte 126, 4 Place Jussieu \\ 75252 Paris CEDEX 05, FRANCE \\ Geoffrey Harris and Enzo Marinari[‡] \\ Physics Department and NPAC \\ Syracuse University \\ Syracuse, NY 13244, USA \\ Emil Martinec \\ Enrico Fermi Institute and Department of Physics \\ University of Chicago \\ Chicago, IL 60637, USA \\ Marco Picco \\ Dipartimento di Fisica, \\ Università di Roma Tor Vergata \\ Viale della Ricerca Scientifica \\ 00133 Roma, Italy.
}

April 1993 


\begin{abstract}
We analyze the behavior of the ensemble of surface boundaries of the critical clusters at $T=T_{c}$ in the $3 d$ Ising model. We find that $N_{g}(A)$, the number of surfaces of given genus $g$ and fixed area $A$, behaves as $A^{-x(g)} e^{-\mu A}$. We show that $\mu$ is a constant independent of $g$ and $x(g)$ is approximately a linear function of $g$. The sum of $N_{g}(A)$ over genus scales as a power of $A$. We also observe that the volume of the clusters is proportional to its surface area. We argue that this behavior is typical of a branching instability for the surfaces, similar to the ones found for non-critical string theories with $c>1$. We discuss similar results for the ordinary spin clusters of the $3 d$ Ising model at the minority percolation point and for $3 d$ bond percolation. Finally we check the universality of these critical properties on the simple cubic lattice and the body centered cubic lattice.
\end{abstract}

PACS numbers: $05.50,11.17,11.00,68.00,64.00$

hep-th/9304088

EFI 93-24

SU-HEP-4240-531

LPTHE 93-20

ROMA2/93/14 
It is a long-standing hope in theoretical physics that it is possible to find a formulation of three-dimensional phase transitions dual to the usual order parameter field theory. Such a description recasts the dynamics in terms of fluctuating surfaces. In the $3 d$ Ising model such a reformulation is indeed possible on the lattice [1], and the relevant problem is the existence of a continuum limit for the surface theory. Despite much theoretical effort, real progress in this direction has been slow and difficult. In order to provide some experimental data to perhaps point in the right direction, we embarked on a numerical study of surfaces in the $3 d$ Ising model.

The main obstacle to a continuum surface description is the well-known fingering instability of surfaces in embedding space of dimension $d>2[2]$. For $d \leq 2$ the theory of random surfaces is solved[3], providing a striking confirmation of the scaling predictions of Liouville theory[4]. Another motivation for our study was to see if the $d>2$ instability plagues Ising surfaces, and to look for scaling properties in the distribution of surfaces of fixed genus. We will present data that shows nontrivial topology-dependent scaling behavior for self-avoiding surfaces in $3 d$ (for previous work, see [5]).

Contrary to the widely held belief that a phase transition is characterized by nested clusters of ordered domains of all possible sizes, it has been shown [6] that the distribution of domain boundaries in the $3 d$ Ising model does not scale at criticality. Rather, as the critical point is approached from low temperatures, islands of flipped spins (which we shall call minority clusters) merge into a large percolating cluster at a temperature $T_{p}$ well below the critical temperature $T_{c}$. The dynamics is then dominated not by the entropy in the cluster distribution but by the entropy of configurations of the percolating cluster. Moreover, no local order parameter of the Ising model reflects this percolation transition.

However there exists a cluster representation of the Ising model due to Fortuin and Kasteleyn [7] which captures the critical properties of the model, in particular the divergence of the correlation length is related to the percolation of the FK clusters. These clusters are formed by adjoining neighboring spins with a probability $1-e^{-2 \beta}$ if they are equal. The FK representation led Swendsen and Wang to propose a Monte Carlo that partially defeats critical slowing down [8]. In our simulations we have used the SW algorithm. We studied the self-avoiding surfaces bounding both minority spin clusters and FK clusters. To explore further the possible realizations of self-avoidance, we also simulated the surfaces defined by pure bond percolation [9]. It should be 
emphasized that the 'bosonic' surfaces defined by Ising domain walls are not the same as the 'fermionic' surfaces that arise in the surface reformulation of the lattice Ising model; nevertheless we expect that they should capture the characteristic features of any critical surface theory of the $3 d$ Ising model.

We ran a medium sized simulation, using roughly four months of time on RISC workstations. We have analyzed Ising configurations on a $64^{3}$ body centered cubic (BCC) lattice at a temperature of $\beta=.0857$ using $.3 \times 10^{6}$ iterations. We also collected data on simple cubic lattices of size $32^{3}$ and $64^{3}$ at $\beta=0.221651$ (performing about $6 \times 10^{6}$ and $.25 \times 10^{6}$ iterations respectively). Data were also taken at the minority percolation point for the Ising model (where we also studied many different lattice sizes, going from $32^{3}$ to $100^{3}$ ) and for pure bond percolation on the simple cubic lattice. All of our error analysis has been done by using jack-knife and binning techniques. For more details see ref. [10].

On the BCC lattice, we coupled with equal strength both the 6 nearest and 8 next-nearest Ising spins so that only three plaquettes of the dual lattice meet along a dual link. Since surfaces built this way on the BCC lattice are naturally self-avoiding, computing the genus of the dual surface is trivial. The number of handles $g$ is obtained through the Euler formula $2-2 g=$ $V-L+P$, where $V, L$, and $P$ are the number of vertices, links, and plaquettes, respectively on the dual surface. On the other hand, the genus definition on the simple cubic lattice is more problematic, and requires a few choices (which we discuss in detail in ref. [10]) to resolve ambiguities where surfaces self-touch.

Overall, for the FK clusters we obtained our best results on the BCC lattice. We found a scaling law for the number of clusters of volume $V_{c l}$, $N\left(V_{c l}\right) \sim V_{c l}^{-\tau}$; the exponent $\tau=2.22$ [11] with a large systematic error which could be as high as $3 \%$ [10]. We also measured the quantity $A_{c l}$, which counts the number of cluster sites on the boundary, and found that asymptotically it was proportional to the cluster volume $V_{c l}$.

Fig. 1 shows this dependence on the simple cubic lattice. We see that for very small volumes, the lattice regularization constrains $V_{c l}$ to equal $A_{c l}$ and for intermediate volumes, there is a small deviation from linear scaling (as some interior sites begin to appear). The plateau that appears around $V_{c l}=3000$ indicates the onset of scaling regime where $A_{c l} \propto V_{c l}$. The growth just at the end of the plot is due to the largest cluster, which wraps around the lattice and merges with itself to form extra interior points. This plateau is the 
first indication that the surfaces are not smooth and are unstable towards the formation of quasi-one-dimensional objects. The observed proportionality of $V_{c l}$ and $A_{c l}$ is well-known in the context of pure percolation in 2 and 3 dimensions [9]. We note that in the well understood case of the $2 d$ Ising model (which has a non-pathological continuum behavior) the cluster perimeter is not proportional to the area spanned by the cluster. The area-volume proportionality, along with the relation [11] to the magnetization exponent $\delta=1 /(\tau-2)$, implies that the scaling behavior of these 'polymers' are related to critical properties of the Ising model.

Turning to the analysis of the topology of the dual surfaces bounding the clusters, we consider the distribution $N_{g}(A)$, where $g$ is the genus and $A$ is the dual surface area. In Fig. 2, we present our data for genus 5 along with a best fit to the functional form

$$
N_{g}(A)=C_{g} A^{x(g)} e^{-\mu(g) A} .
$$

The fit is superb. One of our main results is that this functional form fits our data very well for $g \geq 2$ up to about $g=20$ where our statistics become poor. The 'cosmological constant' $\mu$ is found to be independent of the genus for $g>2$ (Fig. 3 ). The value $\mu^{-1}=114 \pm 3$ is proportional to the average surface area (in lattice units) per handle.

In Fig. 4 we plot the exponent $x(g)$ as a function of the genus. Once again after a transient region for small genus $(g=0-4)$ we find an almost linear behavior in the region $g=5-15$ with a slope of $1.25 \pm 0.1$. The deviations from linearity are small, and in our observation window they can be fitted with an effective exponent correction of order 0.1 , or with logarithmic corrections. These make an estimate of the large genus behavior of $x(g)$ rather difficult. The region we are observing is still transitory, and we cannot exclude that the asymptotic slope of $x(g)$ could equal one at large $g$. This value would be expected if the handles were completely uncorrelated as for a Poisson distribution $P_{g}(A)=\frac{1}{g !}(\mu A)^{g} e^{-\mu A}$.

The results presented above have also been analyzed for the simple cubic lattice. The data agree with those of the BCC and point to a good universal behavior. The main discrepancies can be traced to the short distance ambiguities which plague the definition of the dual surfaces on the simple cubic lattice. The scaling exponents are close for the two lattices. The difference among non-universal quantities, e.g. $\mu^{-1} \sim 60$ on the simple cubic lattice, can 
be understood from the ratio of the number of plaquettes of the respective Wigner-Seitz cells.

We will discuss in detail in [10] the analogous features for both the percolation of minority spins of the Ising model and for non-interacting bond percolation. The general picture is interestingly the same; indeed, the slope $d x / d g$ for bond percolation is compatible with the value for FK clusters. However, the slope $d x / d g \sim 0.7 \pm 0.1$ in the range $g=3-40$ for Ising minority spin percolation. The same caution as before apply to these slope values. Another interesting result is the similarity of the measured area scaling exponents ( $\tau=2.18 \pm 0.05$ upon extrapolation to large lattices) as well as the linear relation between cluster volume and area at the respective critical points (FK clusters at $T_{c}$ and minority spin clusters at $T_{p}$ ). The difference of the $x(g)$ estimated for the two interacting theories we are studying is interesting. It is possible that in the asymptotic region one will get 1 for the slope in both cases, but the large difference of the measured exponents (which are, on the contrary, very similar when looking at FK clusters and at bond percolation) says at least something about finite size corrections, i.e. about the nature of the interaction. Apart from this effect these results indicate that the cluster distribution scaling is rather insensitive to the average cluster density, which is about ten times less for minority spin clusters. At least partially we may be observing some universality of different definitions of self-avoiding random surfaces.

We regard the outcome of our topological studies and the behavior $V_{c l} \sim$ $A_{c l}$ as a strong indication that the cluster boundaries are in a 'branched' phase. The topological evidence suggests that the surfaces grow fingers which reconnect with a fixed probability per unit area. The cosmological constant of the surfaces of fixed genus is nonzero at the critical point. Adjusting the temperature away from criticality will only increase $\mu$, as the large surfaces are exponentially suppressed (apart from a few surfaces of the size of the lattice above the percolation threshold). Therefore there is no relevant parameter in the theory that could be tuned to allow large surfaces of low genus. One can imagine that some additional parameter (e.g. one that couples to the Euler density of the lattice surfaces, which depends on all the spins in a fundamental cell) could be fine-tuned to multicriticality (for another approach see [12]). Then there would be the possibility to have a scaling theory at fixed genus. However, such a tuning will probably not remove the fingering instability of the surfaces, so we still are faced with the problem that the 
continuum theory is not a theory of surfaces but of quasi-one-dimensional objects.

The approximate linearity of $x$ with $g$ is strongly reminiscent of the scaling behavior of $d \leq 2$ random surfaces ${ }^{1}$. But note however that similar scaling behavior can be shown in the 'double-scaling' limit of $O(N)$ vector field theories [13], so a similar exponent exists for the scaling of random graphical networks of fixed topology.

Finally, in order to get a more accurate description of the geometry of the minority clusters, we analyzed the distribution of cluster cross sections as a function of their perimeters. Below the critical temperature $T_{c}$ the distribution drops off at a scale of the order of a few lattice spacings, providing further indication that the surfaces are composed of small, highly interconnected tubes. Remarkably, around $T_{c}$ (recall that the surfaces have long since percolated at $T_{p}<T_{c}$ ) we find very good scaling behavior which must be entirely dominated by the cross sections of the large percolated cluster. If one were bold enough to view the percolated cluster as describing the time evolution of interacting strings, one could speculate that this behavior is reminiscent of a Hagedorn-type transition.

We would like to thank Stephen Shenker for essential discussions which led to our investigations. We are also grateful to NPAC for their crucial support. After this work was completed, we received an interesting paper by M. Caselle, F. Gliozzi and S. Vinti (hep-th 9304001) which puts forward ideas related to those presented here. This work was supported in part by the Dept. of Energy grants DEFG02-90ER-40560, DEFG02-85ER-40231, the Mathematical Disciplines Institute of the Univ. of Chicago, funds from Syracuse Univ., by the Centre National de la Recherche Scientifique, by INFN and the EC Science grant SC1*0394.

\footnotetext{
${ }^{1}$ The typical scaling observed for these $d \leq 2$ random surfaces is in the free energy, describing a single fluctuating surface. Our Ising configurations, however, consist of a collection of interacting surfaces. The scalings we observe are for an ensemble of surfaces and not the free energy.
} 


\section{References}

[*] On leave from the Landau Institute of Physics, Moscow

[†] Laboratoire associé No. 280 au CNRS.

[†] Also at Dipartimento di Fisica and INFN, Università di Roma Tor Vergata, Viale della Ricerca Scientifica, 00133 Roma, Italy.

[1] E. Fradkin, M. Srednicki and L. Susskind, Phys. Rev. D21, 2885 (1980); C. Itzykson, Nucl. Phys. B210, 477 (1982); A. Casher, D. Fœrster and P. Windey, Nucl. Phys. B251, 29 (1985); Vl. Dotsenko and A. Polyakov, in Advanced Studies in Pure Math. 15 (1987).

[2] B. Durhuus, J. Frohlich and T. Jonsson, Nucl. Phys. B240, 453 (1984); J. Ambjorn, B. Durhuus, J. Frohlich and P. Orland, Nucl. Phys. B270, 457 (1986); M. E. Cates, Europhys. Lett. 8, 719 (1988).

[3] See for example O. Alvarez, E. Marinari and P. Windey, Random Surfaces and Quantum Gravity, (Plenum, New York USA 1992) and references therein; F. David, Simplicial Quantum Gravity and Random Lattices, to be published; P. Ginsparg and G. Moore, Lectures on $2 d$ Gravity and $2 d$ String Theory, to be published.

[4] K. G. Knizhnik, A. M. Polyakov and A. B. Zamolodchikov, Mod. Phys. Lett. A3, 819 (1988); F. David, Mod. Phys. Lett. A3, 1651 (1988); J. Distler and H. Kawai, Nucl. Phys. B321, 509 (1989).

[5] M. Karowski and H.J. Thun, Phys. Rev. Lett. 54, 2556 (1985); R. Schrader, J. Stat. Phys. 40, 533 (1985).

[6] A. Coniglio and W. Klein, J. Phys. A13, 2775 (1980).

[7] C.M. Fortuin and P.W. Kasteleyn, Physica 57, 536 (1972).

[8] R.H. Swendsen and J.-S. Wang, Phys. Rev. Lett. 58, 86 (1987).

[9] D. Stauffer and A. Aharony, Introduction to Percolation Theory, (Taylor and Francis, London, U.K. 1992). 
[10] V. Dotsenko, G. Harris, E. Marinari, E. Martinec, M. Picco and P. Windey, in preparation.

[11] J.-S. Wang, Physica A161, 149 (1989).

[12] F. David, Europhys. Lett. 9, 575 (1989).

[13] J. Zinn-Justin, Phys. Lett. B257, 335 (1991); P. Di Vecchia, M. Kato and N. Ohta, Nucl. Phys. 357B, 495 (1991); S. Nishigaki and T. Yoneya, Phys. Lett. B268, 35 (1991). 
Figure 1: $\ln \left(V_{c l} / A_{c l}\right)$ vs. $\ln \left(V_{c l}\right)$ for FK clusters on the $L=64$ SC lattice.

Figure 2: The number of genus 5 surfaces as a function of dual surface area $A$ for FK clusters on the $L=64$ BCC lattice, with a best fit to the functional form given in equation 1.

Figure 3: The dependence of the cosmological constant $\mu(g)$ on genus for FK clusters on the $L=64$ BCC lattice.

Figure 4: The dependence of the exponent $x(g)$ (extracted from the fits to equation 1) on genus for FK clusters on the $L=64 \mathrm{BCC}$ lattice. 


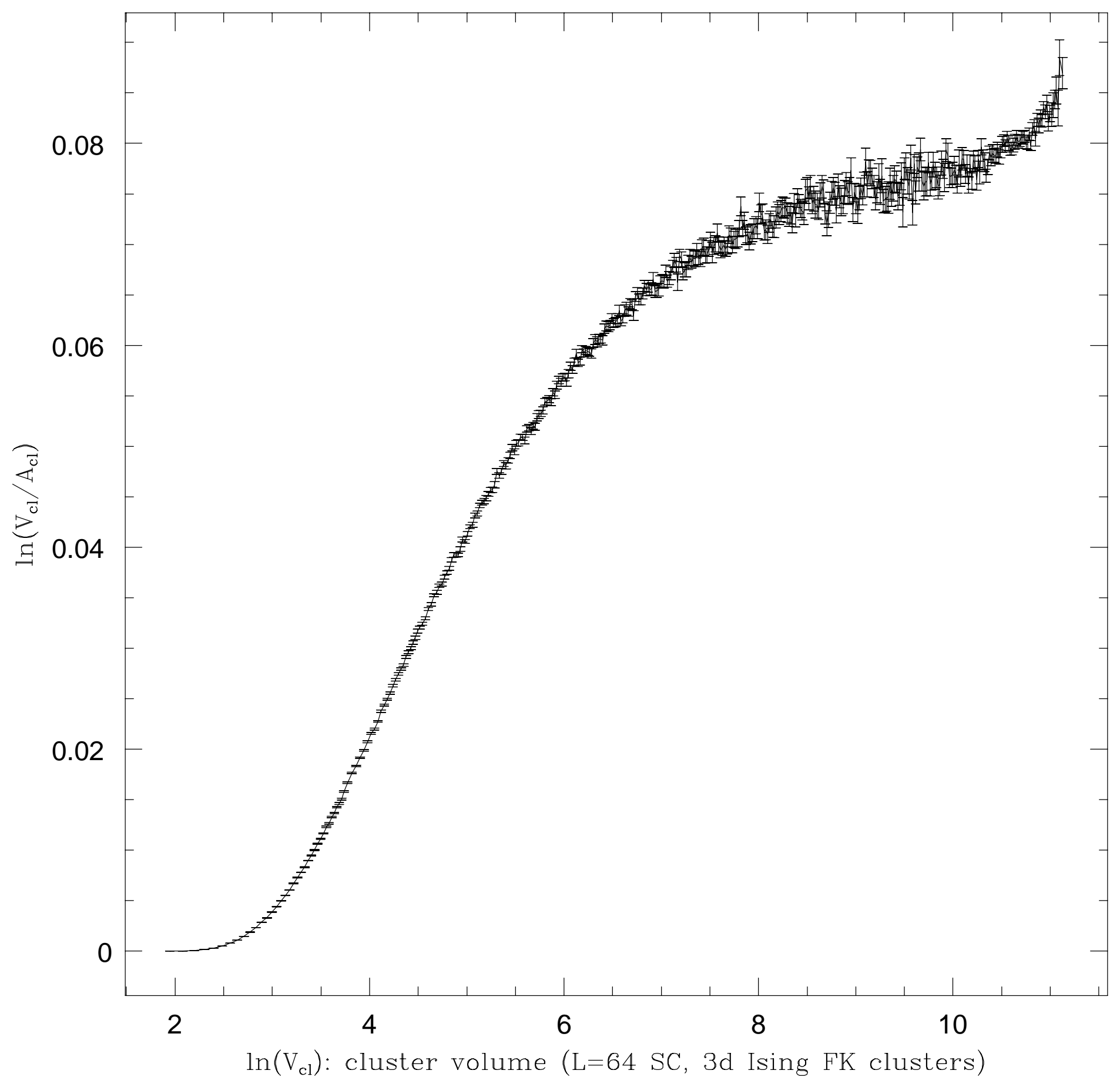




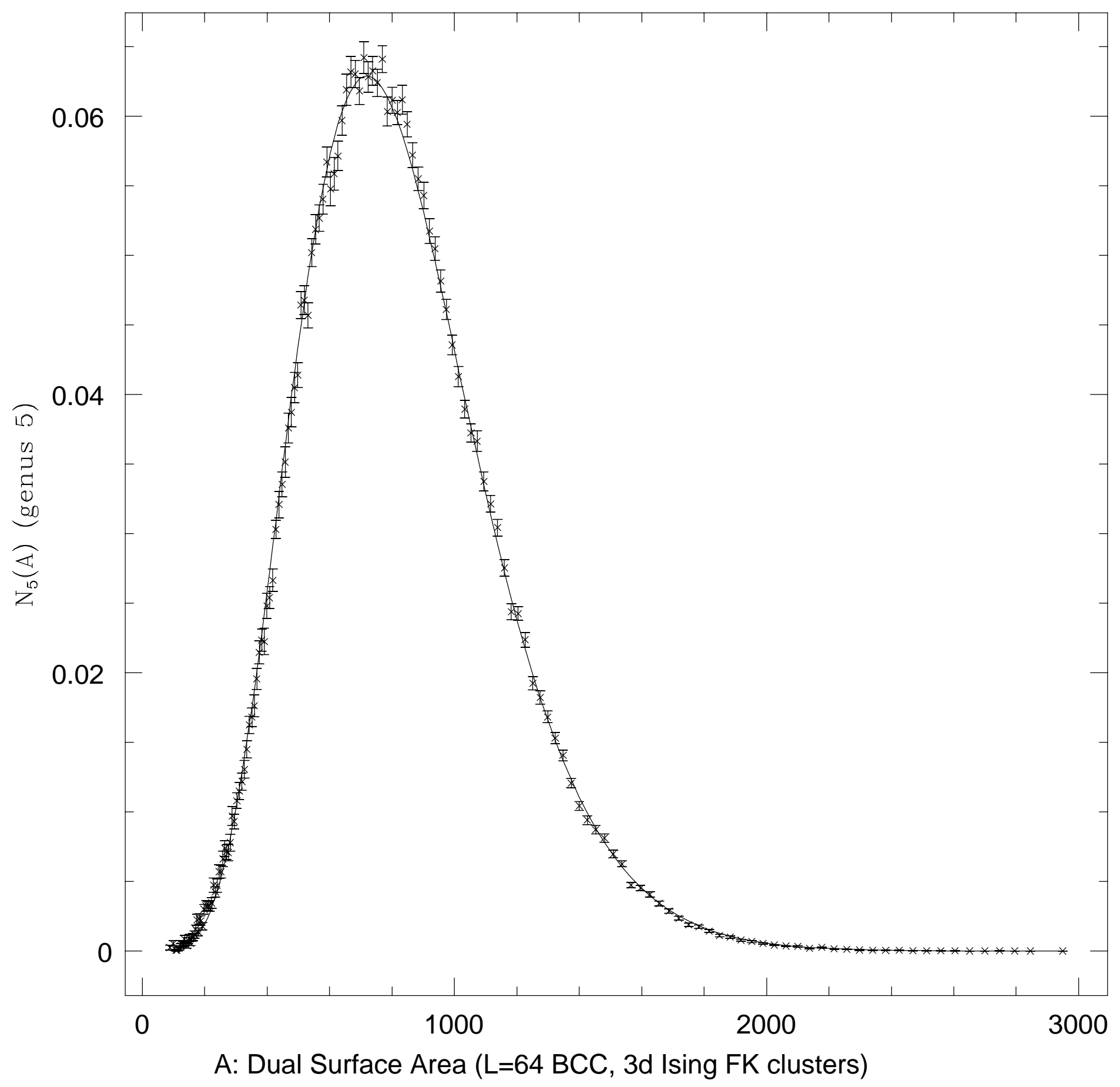




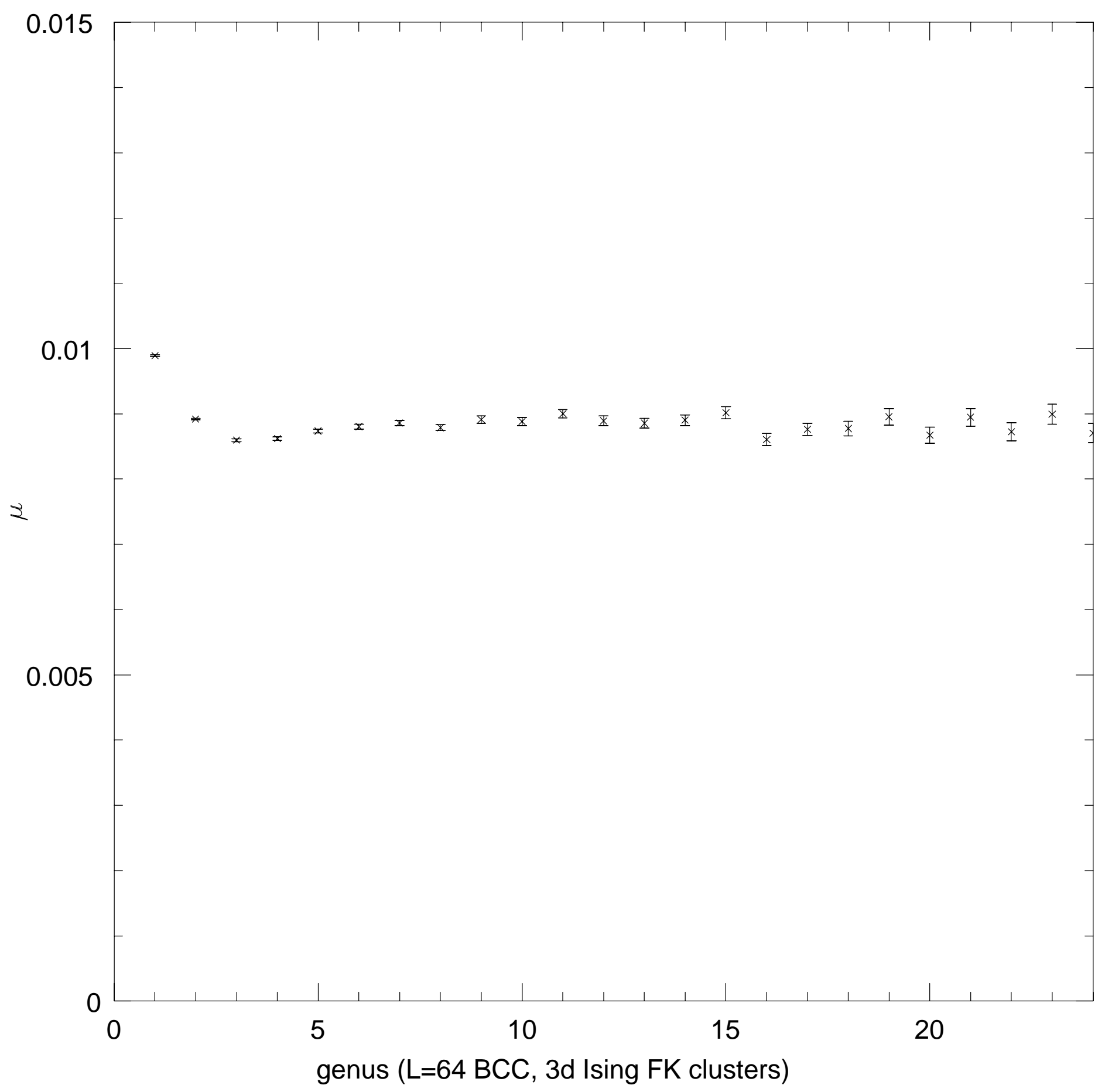




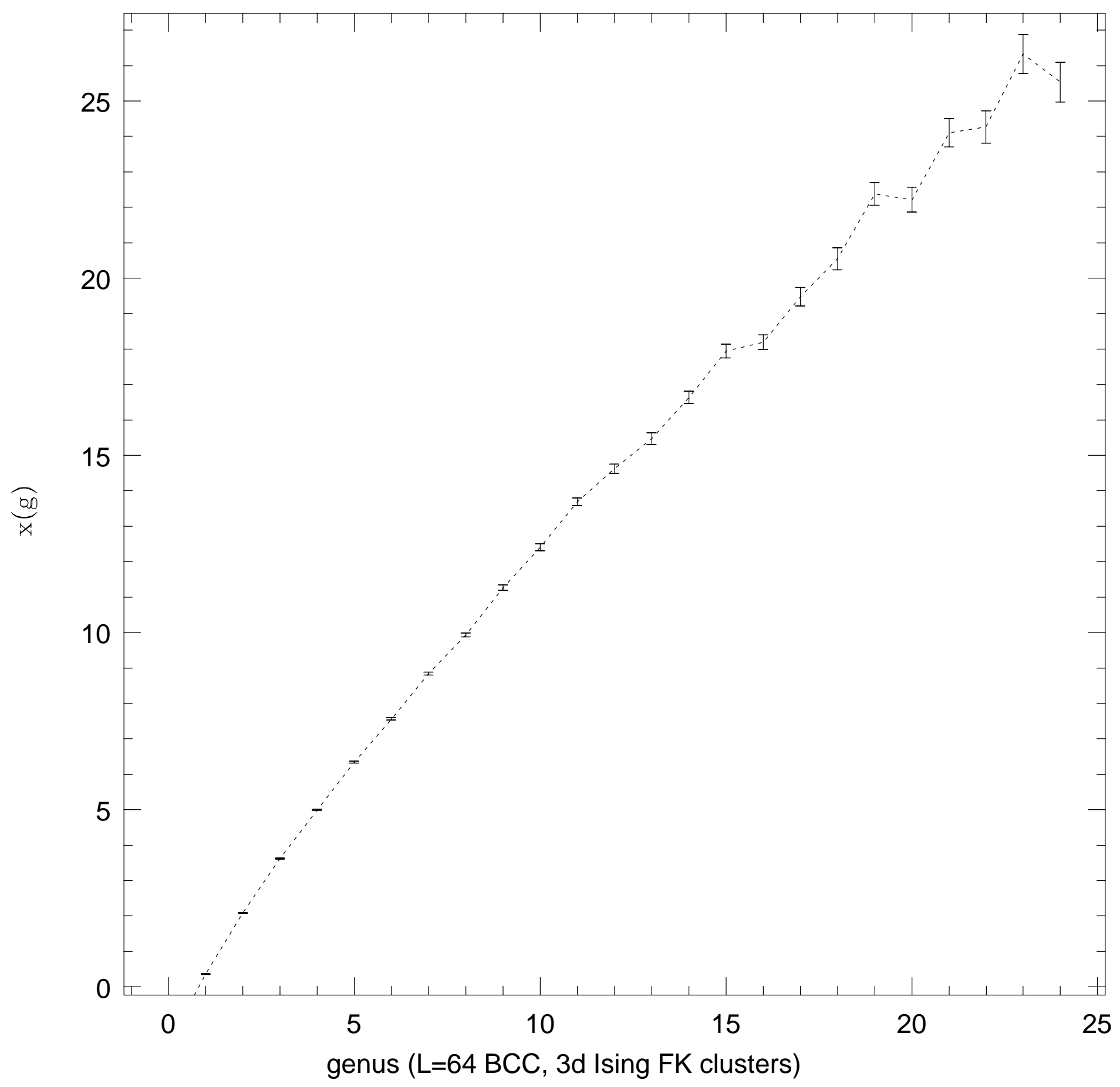

